\title{
Mathematical model of an off-grid hybrid solar and wind power generating system
}

\author{
M. Blasone ${ }^{1}$, F. Dell'Anno ${ }^{2}$, R. De Luca ${ }^{1, \mathrm{a}}$ and G. Torre ${ }^{1}$ \\ ${ }^{1}$ Dipartimento di Fisica "E. R. Caianiello" Università degli Studi di Salerno, Fisciano (SA), Italy \\ ${ }^{2}$ INFN, Sezione di Napoli, Gruppo Collegato di Salerno, Fisciano (SA), Italy
}

\begin{abstract}
The dynamics of an off-grid power generating system, coupled to a storage unit and to household appliances, is described by means of an analytic hydrodynamic analog. Following this analogy, by noticing that the efflux rate from a leaking bucket is described, in terms of the liquid content, by Torricelli's formula, we denote as "Torricelli's smart consumer" a user being able to calibrate its energy consumption rate with respect to the energy level in the storage unit as if the hydrodynamic model would strictly apply. Simple solutions to the nonlinear dynamic problem associated to this type of smart consumer are found and generalization to other types of smart consumers are sought.
\end{abstract}

\section{Introduction}

In the quest for better exploitation of the huge amount of renewable energy available on Earth [1], scientific efforts are nowadays being made in many directions. Solar radiation is being utilized in activating more efficient photovoltaic (PV) cells, in running more complex thermodynamic systems, or in obtaining thermal energy from hybrid PV and thermal (PVT) devices [2]. On the other hand, new and more efficient wind turbines are being designed and fabricated [3]. Hydroelectric plants are being built at smaller scales [4] and geothermal systems are deployed to satisfy heat and electricity needs in various parts of the globe [5]. Biomasses are gathered to run small-scale engines [6] and, finally, the energy of sea waves is being utilized in offshore or coastal power plants [7].

For off-grid users, in particular, a systematic study on the possibility of exploiting locally available renewable energy sources could be useful. In this respect, in a previous work [8] we considered a hybrid wind and solar power generating system, without taking full advantage of the possibility of implementing a feedback between the storage system and the consumer (or "prosumer", as it can be defined a producer and a consumer of electrical energy). In this work, we therefore consider the system, described in Fig. 1, which schematically reproduces the leaking bucket problem [9]: an open container of section $S$ in which a liquid is being poured at an instantaneous flow rate $p(t)$ and from which it flows out at a rate $q(t)$, while the measured liquid level in the bucket is $h(t)$. Even though the flow rate equation (continuity equation) can be generally applied to describe the power produced and dissipated

\footnotetext{
${ }^{\text {a }}$ Corresponding author: rdeluca@unisa.it
}

This is an Open Access article distributed under the terms of the Creative Commons Attribution License 4.0, which permits unrestricted use, distribution, and reproduction in any medium, provided the original work is properly cited. 


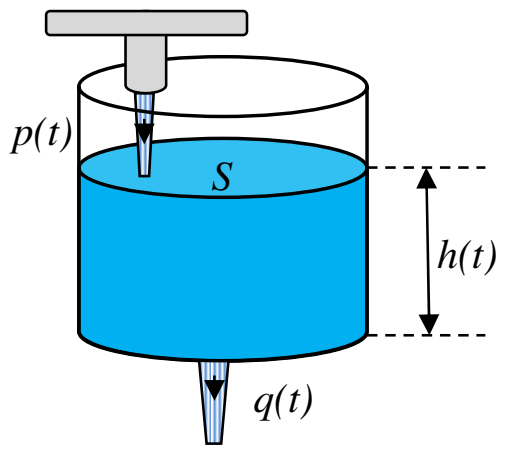

Figure 1. Schematic representation of the "leaking bucket" hydrodynamic system describing a Torricelli's smart consumer. The incoming and outgoing flow rates are $p(t)$ and $q(t)$, respectively. The generated and dissipated electric power are described, in the order, by these two quantities. Furthermore, the energy content in the storage system is described by the height $h(t)$ of the column of liquid in the vessel.

in the off-grid system, our analysis is limited to the strict analogy between the hydrodynamic model and a particular prosumer, denoted as "Torricelli's smart consumer", for which Bernoulli's equations apply [10]. Despite the presence of such drastic limits, the present introductory work may lead to easy generalizations to more realistic systems.

Therefore, a strict analogy between this hydrodynamic model in Fig. 1 and an off-grid electric power generator associated to a storage system and a specific consumer is initially considered. In the following section Bernoulli's theorem and standard methods in solving ordinary differential equations are used to obtain sensible answers to the proposed problem. The effect of small perturbations in the function $p(t)$ on the response of the system are considered in the third section. Finally, in the Conclusions a generalization to different types of consumers (other than Torricelli's smart consumer, for whom the strict hydrodynamic analogy holds) is proposed and further extension of the present work to more realistic cases is suggested.

\section{Torricelli's smart consumer}

Let us consider the hydrodynamic system schematically represented in fig. 1. Write the efflux rate from the bucket as $q(t)=S_{h} \mathrm{v}(t)$, where $S_{h}$ is the area of the hole in the bucket and $\mathrm{v}(t)$ is the velocity of the liquid just below this hole. As already specified, this leaking bucket system can be viewed as an equivalent model of a off-grid electric power generator associated to a storage system and a Torricelli's consumer. In fact, we may consider $p(t)$ as the instantaneous power generated by a hybrid system, which can be any combination of the alternative energy sources listed in the Introduction. The quantity $q(t)$, on the other hand, can be considered to be equivalent to the rate at which household utilities dissipate electrical energy. Finally, the quantity $B(t)=h(t) S$ can be seen as the energy content of a battery used as a storage unit in the off-grid system. As already stated, while the continuity equation can be generally applied to describe power production and dissipation in off-grid systems, the analysis in this Section is limited to the strict analogy between the hydrodynamic model and Torricelli's consumer, for which Bernoulli's equation applies [10]. In this respect, we need to specify that a quasi-stationary fluid flow needs to be assumed. Therefore, when describing the dynamics of the system, we consider the system itself close to its stationary point.

In the simplest formulation of the problem, where the height $h(t)$ is kept constant, Torricelli's formula reads [10]

$$
\mathrm{v}=\sqrt{2 g h}
$$




\section{$3^{\text {rd }}$ European Energy Conference}

so that the consumer's household appliances dissipate energy at a rate $q(t)=S_{h} \sqrt{2 g h}=S_{h} \sqrt{\frac{2 g}{S} B}$, which therefore is seen to depend on the battery energy content $B$, as if the consumer decided, on the basis of the value of $B$, which appliances to use. In this respect, the consumer is defined "smart".

When we allow the height of the liquid to slowly vary, however, we may write the continuity equation as follows:

$$
S \dot{h}(t)+S_{h} \mathrm{v}(t)=p(t)
$$

where the dot stands for "derivative with respect to time". In this way, we may set

$$
\dot{h}(t)=\alpha[f(t)-\mathrm{v}(t)]
$$

where $\alpha=\frac{S_{h}}{S}$ and $f(t)=\frac{p(t)}{\alpha S}$. From Bernoulli's equation written for one point at the top of the liquid column and one just below the opening in the bucket, by considering the fluid to be ideal, we can write

$$
\frac{1}{2} h^{2}(t)+g h(t)=\frac{1}{2} \mathrm{v}^{2}(t) .
$$

Combination of Eqs. (3) and (4) gives

$$
\mathrm{v}^{2}(t)-\alpha^{2}[f(t)-\mathrm{v}(t)]^{2}=2 g h(t),
$$

whose solution provides the generalized Torricelli's formula, for $f(t)=0$,

$$
\mathrm{v}(t)=\sqrt{\frac{2 g h(t)}{1-\alpha^{2}}},
$$

or, for nonzero values of $f(t)$,

$$
\mathrm{v}(t)=\kappa f(t)\left[\sqrt{1+\frac{1}{\kappa}\left(1+\frac{2 \gamma h(t)}{\alpha f^{2}(t)}\right)}-1\right],
$$

where $\kappa=\frac{\alpha^{2}}{1-\alpha^{2}}$, with $0<\alpha<1$, and $\gamma=\frac{g}{\alpha}$. Here, however, $h(t)$ is expressed, in terms of $\mathrm{v}(t)$ and $f(t)$, by Eq. (3), so that Eqs. (6) and (7) are only implicitly determined. Therefore, we need to find an explicit solution for $\mathrm{v}(t)$, which can be obtained as follows.

First, we differentiate Eq. (4) with respect to time, so that

$$
\dot{h}(t) \ddot{h}(t)+g \dot{h}(t)=\mathrm{v}(t) \dot{\mathrm{v}}(t) .
$$

By means of Eq. (4), we next obtain the following ordinary differential equation for the velocity $\mathrm{v}(t)$ :

$$
\dot{\mathrm{v}}(t)=-\kappa \frac{\mathrm{v}(t)-f(t)}{\mathrm{v}(t)+\kappa f(t)}[\dot{f}(t)+\gamma] .
$$

The solution to Eq. (9) thus depends on the particular form of $f(t)$. If $f(t)=f_{0}=p_{0} / \alpha S$ is a positive constant, however, we may easily treat the problem analytically. In fact, the solution to Eq. (9) can be found by separation of variables, by writing

$$
\frac{\mathrm{v}(t)+\kappa f_{0}}{\mathrm{v}(t)-f_{0}} \mathrm{dv}=-\gamma \kappa \mathrm{d} t
$$

In this way, we find:

$$
\mathrm{v}(t)-\mathrm{v}_{0}+(\kappa+1) \ln \left|\frac{\mathrm{v}(t)-f_{0}}{\mathrm{v}_{0}-f_{0}}\right|=-\gamma \kappa t
$$

where $\mathrm{v}_{0}=\mathrm{v}(0)$ is the initial value of the efflux velocity of the liquid from the bucket. Notice that Eq. (11) does not allow an explicit solution for $\mathrm{v}(t)$. Nevertheless, the behavior of the system can be 


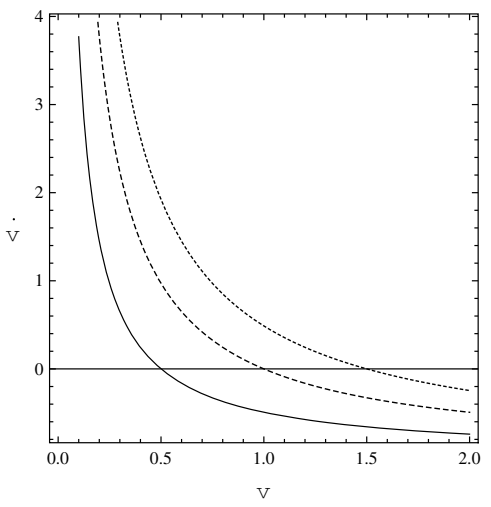

Figure 2. Phase-space representation of the differential equation (10) for $f(t)=f_{0}=0.50 \mathrm{~m} / \mathrm{s}$ (full line), $1.00 \mathrm{~m} / \mathrm{s}$ (dashed line), $1.50 \mathrm{~m} / \mathrm{s}$ (dotted line), and for $\gamma=98.0 \mathrm{~m} / \mathrm{s}^{2}$ and $\kappa=0.101$.

shown by means of a phase-space analysis as in Fig. 2. From this figure we can see that $\mathrm{v}(t)=f_{0}$ is a stable fixed solution, which can be reached from below or from above, depending on the value of $f_{0}$ and $h_{0}$. Therefore, for different choices of parameters, we may see that the implicit solution, provided we consider $\mathrm{v}(t)>f_{0}$ for $\mathrm{v}_{0}>f_{0}$ and $\mathrm{v}(t)<f_{0}$ for $\mathrm{v}_{0}<f_{0}$, can be written as follows:

$$
t=\frac{1}{\gamma \kappa}\left[\mathrm{v}_{0}-\mathrm{v}(t)+(\kappa+1) \ln \left(\frac{\mathrm{v}_{0}-f_{0}}{\mathrm{v}(t)-f_{0}}\right)\right] .
$$

Notice also that, by means of (7), the value of $v_{0}$ can be expressed in terms of the initial value of the height of the liquid $h_{0}=h(0)$ and of $f_{0}$, so that:

$$
\mathrm{v}_{0}=\kappa f_{0}\left[\sqrt{1+\frac{1}{\kappa}\left(1+\frac{2 \gamma h_{0}}{\alpha f_{0}^{2}}\right)}-1\right] .
$$

The condition $\mathrm{v}_{0}>f_{0}$ is summarized by the following simple expression:

$$
h_{0}>\frac{f_{0}^{2}}{2 g}=h^{*} \text {. }
$$

In Figs. 3a and 3b we therefore show, for $\alpha=0.1$ and for two different choices of the $f_{0}$ and $h_{0}$, the numerically determined solution $\mathrm{v}=\mathrm{v}(t)$. In particular, in fig. 3a, we choose $f_{0}=0.10 \mathrm{~m} / \mathrm{s}$ and $h_{0}=1.00 \mathrm{~m}$, so that Eq. (14) holds. In fig. $3 \mathrm{~b}$, on the other hand, we choose $f_{0}=2.0 \mathrm{~m} / \mathrm{s}$ and $h_{0}=0.10 \mathrm{~m}$; in the latter case Eq. (14) does not hold and the fixed point is reached from below. In these figures we may thus notice that the system may reach a stable fixed point either from above (Fig. 3a) or from below (Fig. 3b). The height $h(t)$ can be computed by integrating Eq. (3). In order to understand more deeply the behavior of the system, let us derive the nonlinear ODE for the height of the liquid in the container, so that, by solving with respect to $\mathrm{v}(t)$ in (3) and by substituting into (4), we obtain:

$$
\dot{h}^{2}(t)-\frac{2 \kappa}{\alpha} f(t) \dot{h}(t)+\kappa\left[f^{2}(t)-2 g h(t)\right]=0 .
$$

For $f(t)=f_{0}$, we can thus exhibit the $\dot{h}$ vs. $h$ relation as follows:

$$
\dot{h}=\frac{\kappa f_{0}}{\alpha}\left[1 \pm \sqrt{1+\left(1-\alpha^{2}\right)\left(1-\frac{2 g h}{f_{0}^{2}}\right)}\right]=0 .
$$




\section{$3^{\text {rd }}$ European Energy Conference}
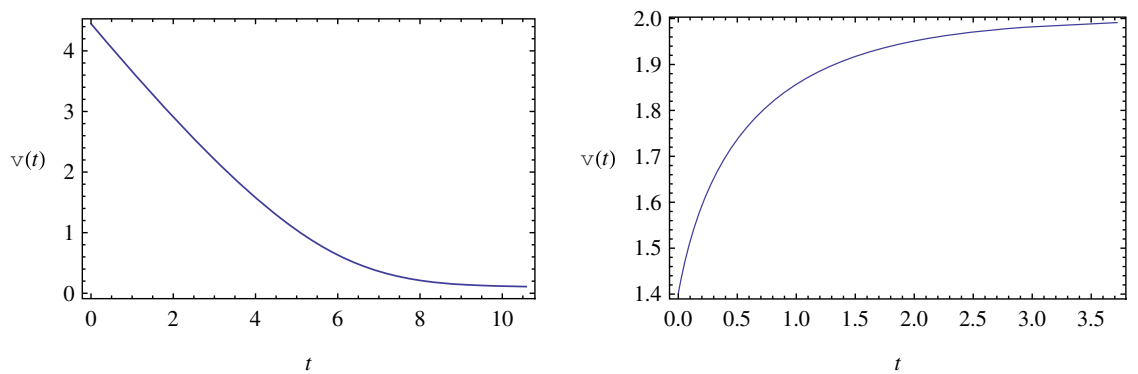

Figure 3. Numerical solution for the efflux velocity $v$ of liquid from the bucket, in the presence of a constant forcing term $f(t)=f_{0}$, for $\alpha=0.1$. In $a$ ) the following choice of parameters is made: $f_{0}=0.10 \mathrm{~m} / \mathrm{s}$ and $h_{0}=1.0 \mathrm{~m}$. In $b$ ), on the other hand, we have set $f_{0}=2.00 \mathrm{~m} / \mathrm{s}$ and $h_{0}=0.10 \mathrm{~m}$. We may notice two different behaviors. In $a$ ) the curve asymptotically goes toward the fixed point $\mathrm{v}^{*}=f_{0}=0.10 \mathrm{~m} / \mathrm{s}$ from above. In $b$ ) the curve approaches the value $\mathrm{v}^{*}=f_{0}=2.0 \mathrm{~m} / \mathrm{s}$ from below.

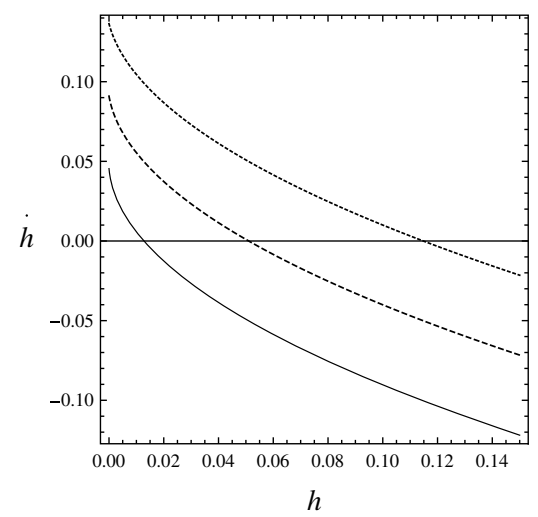

Figure 4. Phase-space representation of the differential equation (16) for $f(t)=f_{0}=0.50 \mathrm{~m} / \mathrm{s}$ (full line), $1.00 \mathrm{~m} / \mathrm{s}$ (dashed line), $1.50 \mathrm{~m} / \mathrm{s}$ (dotted line), and $\kappa=0.101$.

In (16) the minus sign needs to be chosen. The resulting phase-space representation (16) of the nonlinear ODE (15) for $f(t)=f_{0}$ is shown in Fig. 4. We notice that a fixed stable point at $h=h^{*}=\frac{f_{0}^{2}}{2 g}$ is always present. Therefore, we can state that the system has an intrinsic type of intelligence: it always arranges the height of the liquid and the efflux velocity $\mathrm{v}$ in such a way that a stable fixed point is reached. Of course, for very small orifices $(\alpha \rightarrow 0)$, the equilibrium height $h=h^{*}$ goes to infinity and the container needs to be sufficiently high for the system to reach its equilibrium point.

When we refer these results back to the case of a smart consumer who adapts the consumption rate $q(t)=S_{h} \mathrm{v}(t)$ by choosing $\mathrm{v}(t)$ as in Eq. (7), we may restate the above sentences as follows. The off-grid system devised in analogy with the hydrodynamic model studied in the presence of constantly produced electric power presents a stable fixed point for which the energy accumulated does not vary. Therefore, the nonlinear dynamical model (9) predicts the existence of a stable fixed point in which the energy consumption rate adapts to the produced electric power at a given value of the energy content in the storage unit. 


\section{Small perturbations}

In the previous Section we have found an implicit solution for the efflux velocity in a leaking bucket in which liquid is poured at a constant rate. A more general solution for Torricelli's smart consumer's dynamics, can be obtained by solving the nonlinear ordinary differential Eq. (10) in the presence of a slowly varying non-constant forcing term $f(t)$. In this Section we only analyze the effect of small oscillations produced by a forcing term $f(t)=f_{0}+\xi(t)$, where $\xi(t)=\xi_{0} \sin \left(\omega_{f} t\right)$ is a small perturbation, so that $\xi_{0} \ll f_{0}$. This analysis, even though qualitatively already defined in the previous section, allows us to predict the characteristic time in which perturbations in the system decay in time and gives the amplitude of the oscillation about the equilibrium point $h=h^{*}$.

Let us then consider Eq. (15), assuming that, at time $t=0 \mathrm{~s}$, the height of the liquid in the container is at position $h=h^{*}=\frac{f_{0}^{2}}{2 g}$. Starting from $t=0 \mathrm{~s}$, we add a slowly varying oscillatory term $\xi(t)$ to the constant forcing term $f_{0}$. Therefore, on the basis of what said in the previous Section, we expect the forced response of the system to be a function of the type $h(t)=h^{*}+\eta(t)$, where $\eta(t)=\eta_{H}(t)+\eta_{0} \sin \left(\omega_{f} t-\phi\right), \eta_{H}(t)$ being the transient solution and $\eta_{0} \ll h^{*}$. By substituting for the so chosen functions $f(t)$ and $h(t)$ in Eq. (15), we find, by first-order approximation in $\xi$ and $\eta$ :

$$
\dot{\eta}(t)+\frac{\alpha g}{f_{0}} \eta(t)=\alpha \xi(t) .
$$

A general solution to ODE (17) can be found by standard analytical methods [11], so that

$$
\eta(t)=A e^{-t / \tau_{0}}+\frac{\alpha \xi_{0} \tau_{0}}{1+\left(\omega_{f} \tau_{0}\right)^{2}}\left[\sin \omega_{f} t-\omega_{f} \tau_{0} \cos \omega_{f} t\right],
$$

where $\tau_{0}=\frac{f_{0}}{\alpha g}$ and where the constant $A$ can be found by imposing the initial condition $\eta(t)=0$. Besides the transient solution $\eta_{H}(t)$, which decays in the characteristic time $\tau_{0}$, the oscillatory part of (18), which represents the forced solution of the system, is interesting. In fact, this term can be written as $\eta_{0} \sin \left(\omega_{f} t-\phi\right)$, with

$$
\eta_{0}=\alpha \xi_{0} \tau_{0}=\frac{f_{0} \xi_{0}}{g} \quad \text { and } \quad \phi=\tan ^{-1}\left(\omega_{f} \tau_{0}\right)
$$

Therefore, a small oscillatory perturbation in the forcing term, described by the slowly-varying function $f(t)=f_{0}+\xi_{0} \sin \left(\omega_{f} t\right)$, results in a forced oscillatory solution for $\eta(t)$, having amplitude $\eta_{0}$ proportional to $\xi_{0}$. The oscillatory solution $\eta(t)=\eta_{0} \sin \left(\omega_{f} t-\phi\right)$, finally, has a phase shift $\phi=$ $\tan ^{-1}\left(\omega_{f} \tau_{0}\right)$ which, for small values of the argument, i.e. small frequencies $\omega_{f}$, can be seen to be proportional to the characteristic time $\tau_{0}$.

\section{Conclusions}

The properties of a stand-alone electric power generator feeding an energy storage system coupled to a "Torricelli's smart consumer" are studied. The definition of Torricelli's smart consumer comes from the fact that the function $q(t)$, representing the power utilized by this special consumer, is assumed to be equivalent to the flow rate of an ideal liquid pouring out of a leaking container in which the same fluid is stored up to a height $h(t)$. In this hydrodynamic model one assumes that the electric power, generated by exploiting different alternative sources, is equivalent to a function $p(t)$, representing the flow rate of a liquid being poured in the container. By initially taking $p(t)$ to be a constant, we are able to determine some qualitative properties of the system. In fact, by means of Bernoulli's theorem and of the continuity equation, the dynamics of the variable $\mathrm{v}(t)$, representing the efflux velocity from the orifice, and the related dynamics of $h(t)$ are studied by means of a phase-space analysis in the quasi-stationary limit. 
It is found that, for $0<\alpha<1$, the system always presents a stable fixed point. This fact allows us to conclude that the liquid contained in sufficiently high buckets always approaches a fixed height.

Having studied the behavior of the system for a constant value of $p(t)$, we have next allowed small perturbations of amplitude $\xi_{0}$ in this incoming rate function. Therefore, by taking a slowly oscillating perturbation in $p(t)$, we were able to predict its effects on the height $h$ of the liquid. We found that oscillations of amplitude $\eta_{0}=\frac{f_{0} \xi_{0}}{g}$ can be detected, if a first-order perturbation analysis is made.

This type of intelligent consumer, denoted as Torricelli's smart consumer, is strictly related to the hydrodynamic model, which is helpful for describing the dynamics. This simple model, however, limits the validity of our analysis only to a specific type of consumer. In fact, while the continuity equation (2) is a general requirement, even for electrical systems, Bernoulli's equation, which we write as $\mathrm{v}^{2}(t)=\dot{h}^{2}(t)+2 g h(t)$, is specifically valid for ideal fluids. Therefore, only individuals which make use of electrical energy in such a way that the energy consumption rate obeys the law $q(t)=S_{h} \sqrt{h^{2}(t)+2 g h(t)}$, which asymptotically goes to the value $S_{h} \sqrt{2 g h(t)}$, can be denoted as "Torricelli's smart consumers". As future work one may suggest to investigate different types of functional relations between $q$ and $h$. These relations may, eventually, take into account not only the level $h$ of the storage system at time $t$, but may also consider forward predictions and backward feedbacks. Moreover, when dealing only with solar energy, one might introduce an opportune function $p(t)$ taking into account the effective production of energy during the day.

In conclusions, even though the present model is very useful to give a visual representation of how a stand-alone power generating system works, it only allows to predict the overall dynamics in the case a "smart Torricelli's consumer" is the final user. A more general model can thus be envisioned to predict the behavior of a real electrical system. In fact, by specifying the functional relation $q=q(t, h, \dot{h})$, the ODE to be solved for $h$ becomes.

$$
S \dot{h}(t)+q(t, h, \dot{h})=p(t)
$$

where the quantity $h(t) S$ represents the energy content of the storage system. Solution to the above equation depends on the forcing function $p(t)$ and on the particular consumer profile defining the user's daily needs and his intrinsic "smartness". The parameters of the model can be determined by allowing only positive solutions to avoid black-outs at all times. In this way, Eq. (20) can be seen as an energy balance equation according to which the stored energy $h(t) S$ evolves with time, depending on the production rate $p(t)$ and on the controlled consumer function $q=q(t, h, \dot{h})$.

\section{References}

[1] M. Z. Jacobson, M. A. Delucchi, Scientific American, Vol. 301 N. 5, 58 (2009)

[2] T. T. Chow, Applied Energy 87, 365 (2010).

[3] P. Gipe, Wind Power (Chelsea Green Publishing, White River Junction, 2004)

[4] Sachin Mishra, S. K. Singal, D. K. Khatod, Renewable and Sustainable Energy Reviews 15, 3862 (2011)

[5] R. Bertani, Geothermics 34, 651 (2005)

[6] L. Dong, H. Liu, S. Riffat, Applied Thermal Engineering 29, 2119 (2009)

[7] A. F. de Falcão, Renewable and Sustainable Energy Reviews 14, 899 (2010)

[8] M. Blasone, F. Dell'Anno, R. De Luca, G. Torre, Eur. J. Phys. 34, 763 (2013)

[9] J. H. Hubbard, B. H. West, Differential Equations: A Dynamical Systems Approach (Springer, New York, 1991)

[10] F. W. Sears, M. W. Zemansky, H. D. Young, University Physics, $5^{\text {th }}$ ed. (Addison-Wesley, Reading, 1977)

[11] W. E. Boyce and R. C. DiPrima, Elementary differential equations and boundary value problems (Wiley, New York, 1977) 